\title{
XXXIII. Notes on geometrical optics.-Part I
}

\section{Professor Silvanus P. Thompson D.Sc.}

To cite this article: Professor Silvanus P. Thompson D.Sc. (1889) XXXIII. Notes on geometrical optics._Part I, Philosophical Magazine Series 5, 28:173, 232-248, DOI: 10.1080/14786448908621353

To link to this article: http://dx.doi.org/10.1080/14786448908621353

$$
\text { 曲 Published online: } 08 \text { May } 2009 .
$$

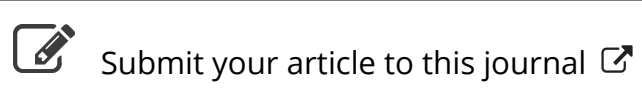

Џلl Article views: 4

Q View related articles ¿ 
XXXIII. Notes on Geometrical Optics.-Part I. By Professor Silvanus P. Thompson, D.Sc.*

\section{The Deduction of the Elementary Theory of Lenses and Mirrors from Wave Principles.}

INTRODUCTORY.-The division of Optics into a " geometrical" part, founded upon the treatment of the subject from the ray point of view, and a "physical" part, founded upon the treatment of the subject from the wave point of view, has long seemed illogical. Experience in the teaching of the science suggests that it would be preferable to adopt the wave theory as a common basis, provided the formulæ of lenses and mirrors which form the staple of geometrical optics are as readily established on wave principles as on ray principles. Five years ago the author of these notes made an attempt to rewrite the elementary part of geometrical optics on wave principles; and though hitherto he has published nothing on the subject, he has subjected the method to the test of experience, and has made it the basis of his optical lectures year by year. The form finally adopted, and now for the first time set forth, has been modified from time to time and simplified. As a result, whilst the formulæ for second approximations, aberration and the like, can be deduced with no greater difficulty than in the orthodox way, the elementary formulæ for first approximations are much more simply deduced, and do not even require the use of trigonometrical functions. This is the more striking because, as all teachers of the subject know, the very first assumptions made in establishing the elementary formulæ of spherieal mirrors and lenses are that angles may be written instead of their sines or tangents. The assumptions made in the method now published are, it is true, different, but involve no greater sacrifice of accuracy, the formulæ being true to the same degree of approximation, and, indeed, though written in a different notation, are in substance identical with those in common acceptance.

It will be convenient, first, to set forth the general bases of the method; next to define the method of reckoning curvatures; then to explain the notation employed; lastly, to develop the elementary formulæ.

\section{General Bases.}

In treating optics from the new standpoint, we have to think about surfaces instead of thinking about mere lines.

* Communicated by the Physical Society; read June 8, 1889. 
Waves march always at right angles to their surfaces; a change in the form of the surface alters the direction of march. The wave-surface is to be considered instead of the "ray." The curvature of the surface therefore becomes the all-important consideration. All that any lens or mirror or any system of lenses or mirrors can do to a wave of light is to imprint a curvature upon the surface of the wave. If the wave is initially a plane wave, then the curvature imprinted upon it by the lens or mirror will result in making it either march toward a point (a real focus) or march as from a point (a virtual focus). If the wave possesses an initial curvature, then all that the lens or mirror can do is to imprint another curvature upon its surface, the resultant curvature being simply the algebraic sum of the initial and the impressed curvatures. As will be seen, in the new method the essential thing to know about a lens or mirror is the curvature which it can imprint on a plane wave : this is, indeed, nothing else than what the opticians call its "power;" the focal power being inversely proportional to the so-called focal length. Another, but less vital point in the method, is the abandonment of the use of the so-called index of refraction in favour of a quantity reciprocally related to it, and here denominated the velocity-constant. The use of the index of refraction dates from a time anterior to the discovery that refraction was a mere consequence of the difference of velocity of the waves in different media. The index of refraction is a mere ratio between the sines (or originally the cosecants) of the observed angles of incidence 'and refraction. The uselessness of clinging to it as a foundation for lens formulæ is shown by the simple fact that, in order to accomplish the very first stage of reasoning in the orthodox way of establishing the formulæ, we abandon the sines and write simply the corresponding angles, as Kepler did before the law of Snell was discovered. The elementary formulx of lenses are, in fact, where Kepler left them. It is now common knowledge that the speed of light, on which refraction depends, is less in optically dense media than in air. The speed of light in air is not materially different from one thousand million feet per second, or thirty thousand million centimetres per second. If we take the speed of light in air as unity, then the numeric expressing the speed in denser media, such as glass or water, will be a quantity less than unity, and will differ for light of different wave-lengths. It is here preferred to take the speed of light in air, rather than in vacuo, as unity, because lenses and optical instruments in general are used in the air. The numeric expressing the 
relative velocity in any medium is called its "velocityconstant"; it is the reciprocal of the index of refraction. The velocity-constant, for mean (yellow) light, for water is about 0.75 ; that of crown glass 0.65 ; that of flint glass from $0 \cdot 61$ to $0 \cdot 56$, according to its density.

\section{Method of Reckoning Curvature.}

The Newtonian definition of curvature as the reciprocal of the radius has a special significance in the present method of treating optics: for some of the most important of lens and mirror formulœ consist simply of terms which are reciprocals of lengths, that is to say of terms which are curvatures. The more modern definition of curvature as rate of change of angle per unit length of the curve (Thomson and Tait's 'Natural Philosophy,'ii. p. 5) is equivalent to Newton's; for if in going along an arc of length $\delta s$, the direction changes by an amount $\delta \theta$, the curvature is $\delta \theta / \delta s$. But the angle $\delta \theta=\delta s / r$, where $r$ is the radius of curvature; hence the curvature $=\delta s / r \delta s=1 / r$.

There is, however, another way of measuring curvature, which, though correct only as a first approximation, is eminently useful in considering optical problems. This way consists in measuring the bulge of the are subtended by a chord of given length.

Consider a circular arc AP, having $\mathrm{O}$ as its centre. Across this are draw a chord $\mathrm{PP}^{\prime}$ of any desired length. The diameter A B bisects it at right angles in M. The short line $\mathbf{M ~ A}$ measures the depth of the curve from arc to chord. If the radius is taken as unity the line $\mathrm{MA}$ is the versed-sine of the angle subtended at B by the whole chord, or is the versed-sine of the semi-angle Fig. 1. subtended at the centre. In Continental works it is frequent to use the name sagitta for the length of this line $\mathrm{MA}$; and as this term is preferable to versed-sine, and can be used generally irrespective of the size of radius, it is here adopted. The proposition is that, for a given chord, the sagitta is (to a first degree of approximation) proportional to the curvature. For it follows from the construction that

assuming PM as unity,

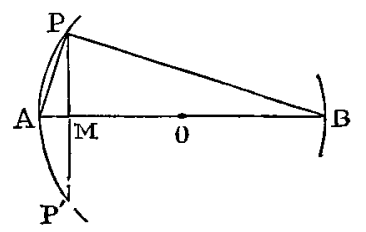

$$
\mathrm{MA}=\frac{1}{\mathrm{MB}}=\frac{1}{2 r-\mathrm{AM}} .
$$


But, for small apertures, AM is small compared with $2 r$, and may be neglected in the denominator, whence

$$
\mathrm{MA} \fallingdotseq \frac{1}{2} \cdot \frac{1}{r} \text {. }
$$

Twice ${ }^{*}$ the sagitta represents numerically the curvature. The error is less than 1 per cent. when the semi-angle subtended at the centre is $10^{\circ}$; less than two per cent. when it is $15^{\circ}$; less than five per cent. when it is $25^{\circ}$.

If the method of reckoning curvatures by means of the sagitta required justification, that is afforded by the fact that the practical method of measuring the curvatures of lenses and mirrors by the spherometer consists essentially in applying a micrometer-screw to measure the sagitta of the arc subtended by a fixed chord, the diameter of the contact circle drawn through the three feet of the instrument. In this case, as indeed in all cases where accuracy, not approximation, is desired, the basis for calculation of the correction exists in the actual size of the diameter of the contact circle, which is a fixed parameter for all measurements made with the instrument.

The sign of the curvature remains to be defined. In the case of actual waves of light, the sign adopted will be + for the curvature of waves which are converging upon a real focus; - for those which are diverging either from a luminous source or from a virtual focus. This agrees with the practice of the ophthalmists and of the opticians, who always describe a converging lens as positive. A positive lens is one which imprints a positive curvature upon a plane wave which traverses it.

The unit of curvature, whether of the wave-surface itself or of the surface of any mirror or lens, will be taken so as to accord with modern ophthalmic and optical practice as the dioptrie; that is to say, the curvature of a circle of one metre radius will be taken as unity. The dioptrie, originally proposed by Monoyer as the unit of focal power of a lens, was formally adopted in $18 \overline{75}$ by the International Medical Congress at Brussels, and its great convenience has led to its universal adoption for the enumeration of the focal powers of lenses. That lens which has a focal length of 1 metre is said to have a focal power of one dioptrie. In other words, such

* Though the sagitta is numerically half the curvature, since all the formulæ of first approximation are homogeneous and of the first degree as regards sagitta and curvatures, the numerical factor $\frac{1}{2}$ disappears in passing from sagittre to curvatures, or vice versâ. 
a lens prints a curvature of one dioptrie upon a plane wave which is incident upon it. For the present proposal to extend the use of the term from focal powers (i.e. imprinted wave-curvatures) to the curvatures of curved surfaces in general, the writer is responsible.

\section{Notation.}

In adopting a notation which embodies the new method it is obviously advisable to choose one which lends itself most readily to the existing and accepted notations. In the great majority of books on optics, the recognized symbol for focal length is $f$; that for radius of curvature $r$. And in the Cambridge text-books for many years the distances from lens or mirror of the point-object and the point-image have respectively been designated by the letters $u$ and $v$. Now it is the reciprocals of these which occur in the expressions for the curvatures of surfaces or of waves. The symbols adopted respectively for the four reciprocals are accordingly $\mathscr{H}, \mathscr{R}, \mathscr{U}$, and $\mathscr{V}$. The accepted symbol for the index of refraction is the Greek letter $\mu$; for the velocity-constant, which is its reciprocal, we take the letter $h$. The following is a tabular statement of the symbols and their meanings:-

\begin{tabular}{|c|c|c|}
\hline Symbol. & Meaning. & $\begin{array}{l}\text { Equivalent in } \\
\text { Current } \\
\text { Notation. }\end{array}$ \\
\hline $\mathscr{H}$ & $\begin{array}{l}\text { Focal eurvature, or Focal power of lens or } \\
\text { mirror }(=\text { dioptries, if metre is taken as unit } \\
\text { of length })\end{array}$ & $\frac{1}{f}$ \\
\hline $\mathscr{R}$ & 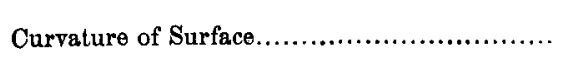 & $\frac{1}{r}$ \\
\hline $\mathscr{U}$ & 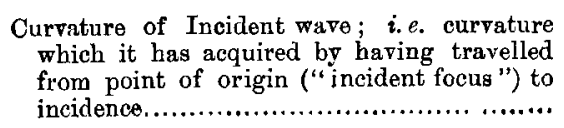 & $\frac{1}{u}$ \\
\hline $\mathscr{\vartheta}$ & $\begin{array}{l}\text { Curvature of Resultant wave; i. e. curvature } \\
\text { with which wave emerges from the lens...... }\end{array}$ & $\frac{1}{v}$ \\
\hline$h$ & 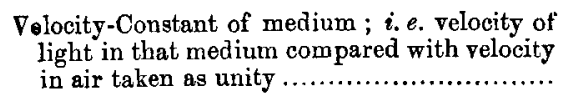 & $\frac{1}{\mu}$ \\
\hline
\end{tabular}

Expansion of Curvatures. - If the curvature $\mathscr{R}$ of a wave at any point is known it is easy to calculate the curvature at 
any other point at distance $d$ further from or nearer to the centre, the formula for the new curvature $\mathscr{R}^{\prime}$ being as follows:-

$$
\mathscr{R}^{\prime}=\mathscr{R} \frac{1}{1 \pm \mathscr{R} d} . \quad . \quad . \quad . \quad . \quad . .
$$

The + sign must be taken where the new point is further from the centre than the point for which the curvature $\mathscr{R}$ is specified; the - sign when it is nearer the centre. This proposition is of use in dealing with thick lenses, and with thin lenses at a given distance apart.

\section{Refraction Fornulae.}

As a preliminary to lens formulæ, it is convenient to consider certain cases of refraction.

\section{A. Prane Surface : Curved Wave.}

Case (i.) a. Entrant Wave of Negative Curvature.

Consider a retarding medium, such as glass, bounded on the left (fig. 2) by a plane surface S S. Let $\mathrm{P}$ be a source of

Fig. 2.

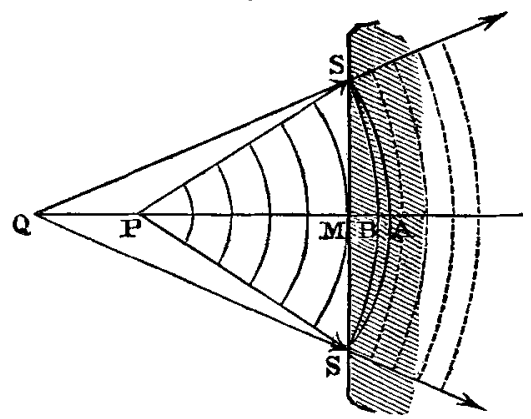

waves incident on the surface, PM being a line perpendicular to SS. The wave-fronts, at successive small intervals of time, are represented by arcs of circles. At a certain moment the wave, had it been going on in air, would have had for its surface the position SAS ; the curvature being measured by the sagitta AM. The medium, however, retards the wave, and it will only have gone as far as B instead of penetrating to $\mathrm{A}$; $\mathrm{B}$ being a point such that $\mathrm{BM}=h . \mathrm{AM}$, where $h$ is the velocity-constant of the medium into which the wave enters. The eurvature of the wave is flattened as the result of the retardation. Now draw a circle through SBS, and find its

Phil. Mag. S. 5. Vol. 28. No. 173. Oct. 1889. 
centre Q. To a first degree of approximation the arc SBS represents the retarded wave-front, the set of wave-fronts from $B$ onwards being represented by the series of ares drawn from $Q$ as centre. An eye situated in the medium on the right of SS will perceive the waves as though coming from $Q$, the (virtual) point-image of $P$. Accurately the wavefronts should be hyperbolic arcs, but if $\mathrm{SS}$ is small relatively to $\mathrm{P} M$ the circular arcs are adequate. Now $\mathrm{A} M=\mathscr{U}$, and $B M=\mathscr{V}$. Hence the action of the plane surface upon the curvature of the incident wave is given by the formula

$$
\mathscr{U}=h \mathscr{U} \cdot \cdot \cdot \cdot \cdot \cdot \cdot
$$

Case (i.) b. Entrant Wave of Positive Curvature.

The entrant wave (fig. 3) has a positive curvature or convergence such as would cause it to march to the point $P$ (the

Fig. 3.

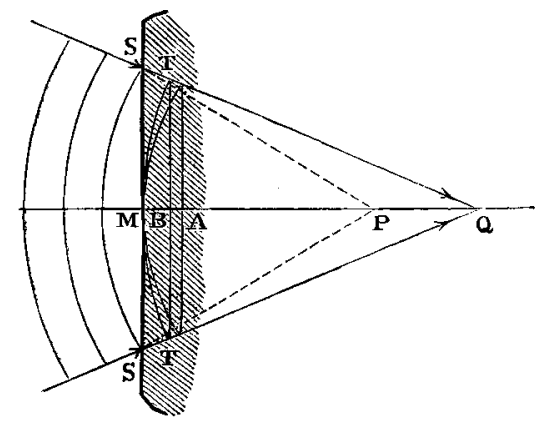

incident focus) if its path lay wholly in air. At a certain moment, when the middle point of the wave-front has reached $\mathrm{M}$, the outer portions of the wave-front passing in through . S S would have reached positions as far as the vertical line drawn through $\mathrm{A}$ had the path lain wholly in air. But being retarded they only reach as far as the line TB T drawn through $\mathrm{B}$; where again $\mathrm{BM}=h . \mathrm{AM}$. The circular arc through T B T has $Q$ for its centre; that is to say, alter entry the waves now converge on $Q$ instend of $P$. In this case also the effect on the wave of entrance into the retarding medium is to flatten its curvature, and the formula is as before,

$$
\mathscr{V}=\hbar \mathscr{U} \cdot \cdot \cdot \cdot \cdot \cdot \cdot(2) \text { bis. }
$$

Case (ii.) a. Emergent Wave of Negative Curvature.

Consider the wave emerging (fig. 4) into air from a point 
$P$, situated in the retarding medium whose velocity-constant is $h$. Had the wave been going on wholly through the denser

Fig. 4.

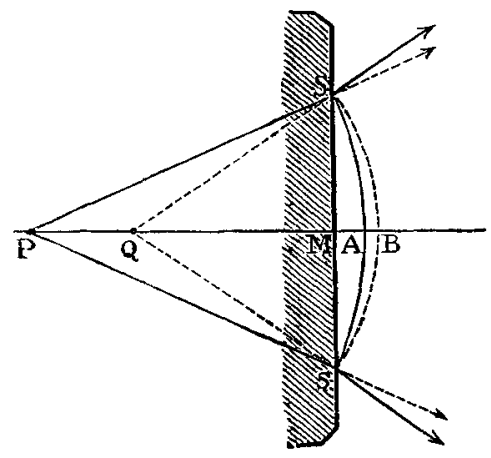

medium, the wave-front would have been at S A S; but, being accelerated on emergence into air, it reaches B instead of $\mathbf{A}$. The new curve S B S has Q for its centre; that is to say, the wave emerges from $Q$ as a virtual focus, its curvature being augmented. The sagitta $B M$ is greater than $A M$ in the ratio of 1 to $h$. Hence in this case the formula is

$$
\mathscr{V}=\frac{1}{h} \mathcal{U} \cdot \ldots . . \cdot .
$$

Case (ii.)b. Emergent Wave of Positive Curvature.

There is no need to prove this case separately; it leads to the same formula

$$
V=\frac{1}{h} \mathscr{U} \cdot \cdot \cdot \cdot \cdot \cdot \cdot(3) \text { bis. }
$$

In the case of either positive or negative initial curvature, emergence from the retarding medium through the plane surface into air augments the curvature.

\section{B. Curved Surface: Plane Wave.}

Case (i.)a. Entrant Wave; Convex Surface.

The surface $S M S$ is convex toward the light; the centre of curvature being $\mathrm{O}$. Consider the plane wave entering the surface. At a certain instant it would have arrived at S A S had its path lain wholly in air. Because of the retarding medium the central portion of the wave-front will only reach $\mathrm{B}$ instead of $\mathrm{A} ; \mathrm{B}$ being such a point that $\mathrm{B} \mathrm{M}=h$. $\mathrm{A} \mathrm{M}$. 
As a result the wave is imprinted with the curved surface S B S, the centre of which is at F, toward which point the Fig. 5.

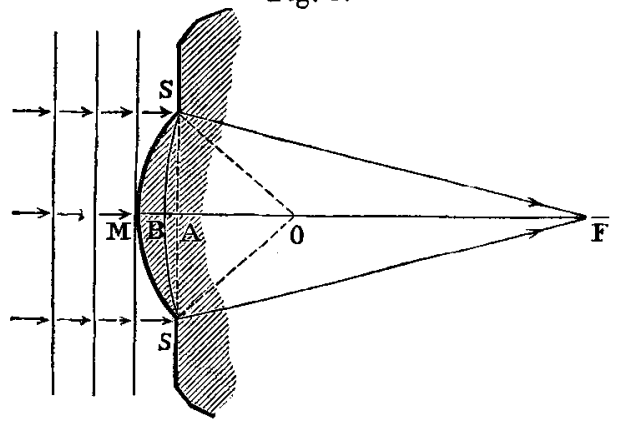

wave converges. The sagitta A B measures the focal curvature thus impressed on the wave. Now

or

$$
\begin{aligned}
\mathrm{A} B & =\mathrm{A} \mathrm{M}-\mathrm{BM} \\
& =\mathrm{A} \mathrm{M}-h . \mathrm{A} \mathrm{M}, \\
\mathscr{H} & =\mathscr{R}(1-h) . .
\end{aligned}
$$

It will be noted that if the curvature of the surface is positive (i.e. bulging toward the source of light), the impressed focal curvature is also positive. For the case of any two media having respective velocity-constants $h_{1}$ and $h_{2}$, the formula becomes

$$
\mathscr{H}=\mathscr{R} \frac{h_{1}-h_{g}}{h_{1}} . . \quad \text {. . . . }
$$

Case (i.) b. Entrant Wave; Concave Surface.

Consider the plane wave which would have reached the Fig. 6.

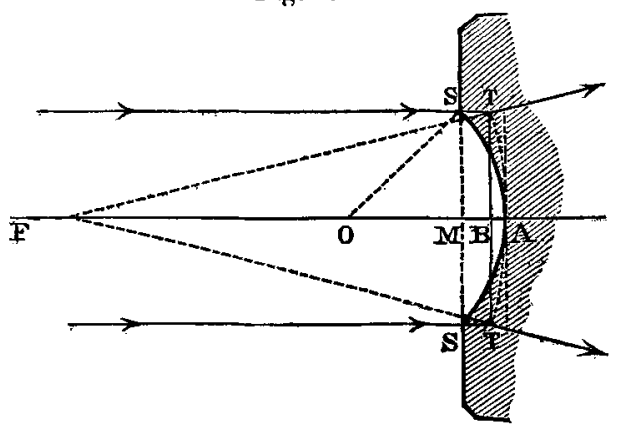

vertical line though A had its path lain wholly in air; the central portion does reach $A$, but the marginal portions are 
retarded, and only reach the vertical line $\mathrm{T} \mathrm{B} \mathrm{T}$ drawn through $\mathrm{B}$, a point such that $\mathrm{B} \mathrm{M}=h$. AM. The impressed curve is T A T with centre F. That is to say, the concave (or negative) surface imprints a negative focal curvature on the wave, its sagitta being $A B$.

$$
\begin{aligned}
\mathrm{A} \mathrm{B} & =\mathrm{A} \mathrm{M}-\mathrm{B} \mathrm{M} \\
& =\mathrm{A} \mathrm{M}-h . \mathrm{A} \mathrm{M}, \\
\mathscr{y} & =\mathscr{R}(1-h) . \quad . \quad \cdot \quad . \quad . \quad \text { (4) bis }
\end{aligned}
$$

The formula, therefore, is the same for entrant plane waves whether the surface be convex or concave, the sign of $\dddot{q}^{\prime \prime}$ following the sign of $\mathscr{R}$.

Case (ii.)a. Emergent Wave; Surface Convex toward light (i.e. concave toward air into which wave emerges).

The plane wave would have had its front at SA S (fig. 7)

Fig. 7.

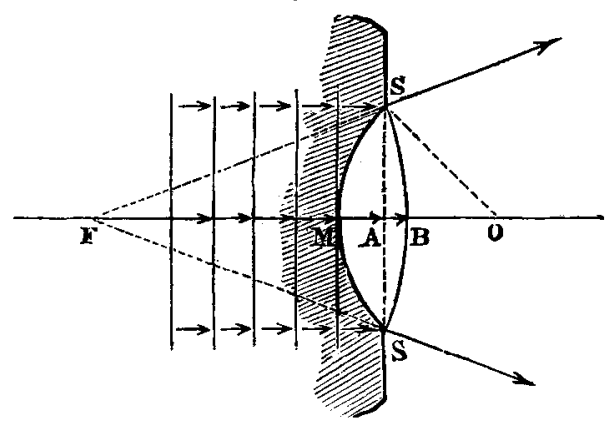

at a certain instant had its path lain wholly in the retarding medium; but the central portion being accelerated by its emergence at $M$ into air reaches $B$, where $B M$ is to $A M$ as 1 to $h$. Hence the curve S B S, whose curvature is measured by the sagitta A B, is the impressed focal curvature. It will be noted that this is of opposite sign to that of the surface of emergence. Hence the sagitta A B must be taken with minus sign.

$$
\begin{aligned}
-\mathrm{AB} & =\mathrm{BM}-\mathrm{AM} \\
& =\frac{1}{h} \mathrm{AM}-\mathrm{AM} \\
+\mathrm{AB} & =\mathrm{AM}\left(1-\frac{1}{h}\right) \\
\mathscr{H} & =\mathscr{R}\left(\frac{h-1}{h}\right) .
\end{aligned}
$$


As before, for any two media having respective velocityconstants $h_{1}$ and $h_{2}$, the formula becomes

$$
\mathscr{H}=\mathscr{R} \frac{h_{1}-h_{2}}{h_{1}}, \quad \cdot \quad \cdot \quad \cdot \quad \cdot \quad \text { (5) bis. }
$$

which, in the present case where $h_{1}<h_{2}$, will give $\not / t$ of opposite sign to $\mathscr{R}$.

Case (ii.)b. Emergent Wave; Surface Concave toward light (i.e. convex toward air into which wave emerges).

This is similar to the preceding, and yields the same formula.

Comparison of Case (i.) a with Case (ii.)b.

Comparing formula (4) with formula (6), we get for the two primary focal curvatures impressed respectively on plane waves passing in opposite directions through the curved surface,

$$
\frac{\mathscr{F}_{1}}{\mathscr{F}_{2}}=-\frac{h_{2}}{h_{1}} ; . \text {. . . . . . }
$$

whence, for the two primary focal lengths,

$$
\frac{f_{1}}{f_{2}}=-\frac{h_{1}}{h_{2}}
$$

The focal lengths are, as the negative sign indicates, to be measured in opposite directions with respect to the surface. Also, taking the algebraic sum of the two primary focal lengths, we get

$$
\begin{aligned}
f_{1} & =r \frac{h_{1}}{h_{1}-h_{2}}, \\
f_{2} & =r \frac{h_{2}}{h_{2}-h_{1}}=r \frac{-h_{2}}{h_{1}-h_{2}}, \\
f_{1}+f_{2} & =r \frac{h_{1}-h_{2}}{h_{1}-h_{2}}=r:
\end{aligned}
$$

and, as one of the focal lengths is negative, it follows that the difference of their lengths is equal to the radius of the curved surface.

\section{Curved Surface : Curved Wave.}

The cases in which a wave possessing initial curvature passes through a curved surface and acquires a resultant curvature may be dealt with, apart from any further geometrical constructions, by applying the principle of super- 
position of curvatures. Thus, take the case of a wave possessing initial curvature $\mathscr{U}$ entering from air into a medium having velocity-constant $h$, and so curved that the focal power of the curved surface is $\mathscr{H}$. Then, as the wave enters the surface of the medium two effects will occur: its initial curvature will be altered in the ratio of the velocities, and there will be superposed upon it the focal currature of the surface; or, in symbols,

$$
\mathscr{V}_{1}=h \mathscr{U}+\mathscr{H}_{1} \cdot \ldots \cdot . \cdot . \cdot
$$

For an emergent wave, possessing initial curvature $\mathscr{U}$ in the medium, the formula will be

$$
\mathscr{V}_{2}=\frac{1}{h} \mathscr{U}+\mathscr{H}_{2} \cdot \ldots . . . .
$$

Or, for the case of a wave passing from a medium of velocityconstant $h_{1}$ to another of velocity-constant $h_{z}$, the formula will be

$$
\mathcal{V}=\frac{h_{2}}{h_{1}} \mathscr{U}+\mathscr{\%} \cdot . . . . . .
$$

It is easy, however, to prove any one of the several cases that may arise, without in this way relying upon the principle of superposition. Take the case of a positive wave enterjng a positively curved surface.

Let SCS (fig. 8) be the surface of the medium, its cur-

Fig. 8.

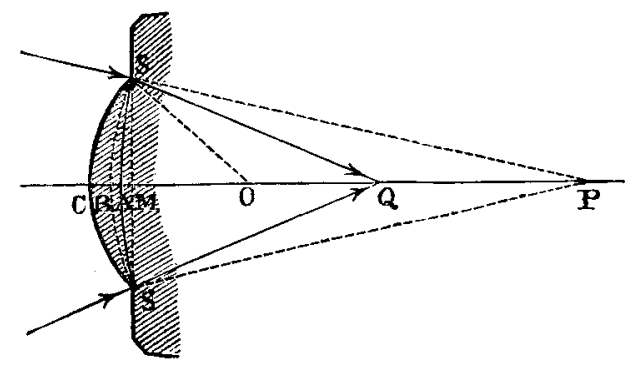

vature being measured by the sagitta $\mathrm{CM}$. There will be a certain moment when the entrant wave, converging toward P, would have had as its front S A S had its path lain wholly in air. But the central portion has entered the retarding medium at $\mathrm{C}$, hence will only have advanced as far as $\mathrm{B}$ instead of $\mathrm{A}$; $\mathrm{B}$ being such that $\mathrm{BC}=h . \mathrm{AC}$. Hence the resultant wave will have the form $\mathrm{SBS}$, and the sagitta of the resultant curvature is $B$ M. 
But

$$
\begin{aligned}
\mathrm{BM} & =\mathrm{CM}-\mathrm{CB}, \\
& =\mathrm{CM}-h . \mathrm{AC}, \\
& =\mathrm{CM}-h(\mathrm{CM}-\mathrm{AM}), \\
& =\mathrm{CM}(1-h)-h \cdot \mathrm{AM} ; \\
\mathscr{V} & =\mathscr{R}-h \mathscr{R}+h \mathscr{U} .
\end{aligned}
$$

whence

$$
\mathscr{R}(1-h)=\mathscr{\%}
$$

$$
\mathscr{V}=\mathscr{s}+h \mathscr{U} \cdot \text {. . . . . (8) bis. }
$$

D. Lens Formule.

Case i. Thin Lens; Plane Wave.

In the case of a lens, the curvature $\mathscr{H}_{1}$ imprinted on a plane wave by entrance at the first surface may be regarded as an initial curvature of the wave which emerges through the second surface. Emergence into air will, as shown above, alter the curvature by augmenting it in the ratio of 1 to $h$, and superpose upon it the focal curvature $\%_{2}$ due to the second surface. Hence the whole resultant curvature $q^{*}$ imprinted by a thin lens on the plane wave will be

$$
\mathscr{\psi}=\frac{1}{h} \mathscr{\%}_{1}+\mathscr{\psi}_{2}
$$

But

$$
\mathscr{H}_{1}=\mathscr{R}_{1}(1-h) \text {, }
$$

and

$$
\mathscr{H}_{2}=-\mathscr{R}_{2}\left(\frac{1-h}{h}\right)
$$

whence

$$
\mathscr{H}=\mathscr{R}_{1} \frac{1-h}{h}-\mathscr{R}_{2} \frac{1-h}{h},
$$

or

$$
\mathscr{F}=\left(\mathscr{R}_{1}-\mathscr{R}_{2}\right) \frac{1-h}{h} . \quad . \quad . \quad .
$$

This formula may be compared with that in the current notation,

$$
\frac{1}{f}=\left\{\frac{1}{r_{1}}-\frac{1}{r_{2}}\right\}(\mu-1) \text {. }
$$

In the case of a lens composed of a medium $h_{2}$, lying between two other media $h_{1}$ and $h_{3}$, the formula becomes

$$
\mathscr{q ^ { \prime }}=\frac{1}{h_{1} h_{2}}\left\{\mathscr{R}_{1}\left(h_{1}-h_{2}\right) h_{2}+\mathscr{R}_{2}\left(h_{2}-h_{3}\right) h_{1}\right\} \ldots
$$


Case ii. Thick Lens; Plane Wave.

If the lens has thickness $d$, the rule for expansion of curvature at end of $\$ 4$ above at once gives

or

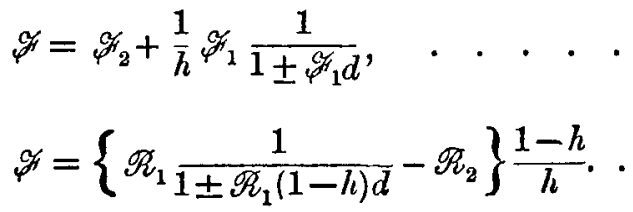

Case iii. Any Lens; Any Wave.

The principle of superposition at once gives the universal formula for all lenses bounded by identical media on the two sides :-

$$
\mathcal{V}=\mathscr{U}+\mathscr{H} ; \cdot \cdot \cdot \cdot \cdot
$$

or, in words, the resultant curvature is the algebraic sum of the initial curvature and the impressed curvature. This may again be compared with the formula in current notation:

$$
\frac{1}{v}=\frac{1}{f}-\frac{1}{u} \text {. }
$$

The difference in sign attributed to the term $\frac{1}{u}$ arises from conventions adopted in the two systems.

E. Two Thin Llenses at distance apart.

The principle of expansion of curvature at once gives us as the equivalent focal power,

$$
\mathscr{H}=\mathscr{H}_{2}+\mathscr{\psi}_{1} \frac{1}{1+\mathscr{\psi}_{1} d}, \ldots . . .
$$

where $\mathscr{\psi}_{1}$ and $\mathscr{\psi}_{2}$ are the focal powers of the first and second lenses, and $d$ the distance between them. $\mathscr{F}$ will be in dioptries if $\mathscr{\%}_{1}$ and $\mathscr{\%}_{2}$ are in dioptries and $d$ in metric units. If the two thin lenses are close together, the resultant power is simply the algebraic sum of the powers of the separate lenses. One simply adds the dioptries of the separate lenses to find the resultant dioptries.

\section{Reflexion Formula. \\ Mirrors.}

Case i. Plane Mirror; Curved Wave.

The mirror (fig. 9) has surface SM S. The incident wave would have had front $S$ A S at a certain instant had its path lain 
wholly in air. The central portion of the wave, which would have reached $A$, travels backwards to $B$, an equal distance, in

Fig. 9.

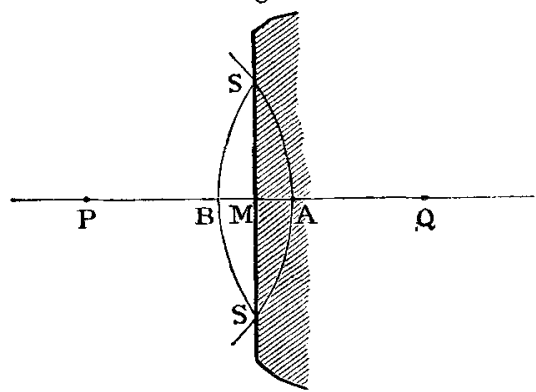

the same time. The sagitta $B M$ of the resultant curvature is equal to and of opposite sign to the sagitta $\mathrm{AM}$ of the initial curvature ; or

$$
\mathscr{V}=-\mathscr{U} \cdot \cdot \cdot \cdot \cdot
$$

Case ii. Curved Mirror; Plane Wave.

There are two cases, equally simple, of convex and concave mirrors. One will suffice. Consider (fig. 10) a plane wave which at a certain instant would have arrived at SAS had its path lain wholly in air. The central portion of the wave has, however, struck at $\mathrm{M}$, and marches backwards to $\mathrm{B}$ in same time as it would bave taken to reach $A$. Hence

or

$$
\mathrm{BM}=\mathrm{AM} \text {, }
$$

$$
\mathrm{BA}=2 \mathrm{AM} \text {. }
$$

But A M measures the curvature of the mirror, whilst BA measures

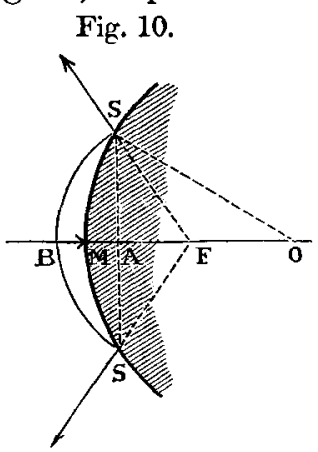
the curvature impressed on the plane wave. Hence

$$
\mathscr{H}=2 \mathscr{R} . \cdot \cdot \cdot \cdot \cdot \cdot
$$

Case iii. Any Mirror ; Any Wave.

The principle of superposition at once leads to a general formula, expressing the sum of the two actions of the mirror on the wave; it reverses its initial curvature, and then imprints a focal curvature upon it. In symbols,

$$
\vartheta=-\mathscr{U}+\mathscr{H} \cdot \ldots . . .
$$




\section{Formula of the Lenticular Mirror.}

The lenticular mirror consists of a lens one face of which is silvered, so that the light passes twice through the lens, and suffers an intermediate reflexion at the silvered surface. If the focal power of the lens be $\mathscr{H}_{1}$, and the curvature of the mirror-surface be $\mathscr{R}$, then the total curvature which the system impresses upon a plane wave will be equal to $2 \mathscr{\psi _ { 1 }}+2 \mathscr{R}$, and there will be a reversal of the direction of the light, so that

$$
\mathscr{H}=-2\left(\mathscr{H}_{1}+\mathscr{R}\right) \text {. . . . . . }
$$

\section{A Dioptrie Spherometer.}

In order the better to bring to practical issues the method of treating lens problems by curvatures, the author designed a special spherometer. In this instrument the readings of the scale are caused to indicate directly the dioptries of the curvature; that is to say, one whole turn of the micrometric screw corresponds accurately to a curvature having one metre as its radius. In the particular instrument now exhibited the pitch of the micrometric screw is 1 millimetre. To make this correspond to a curvature of one dioptrie, the radius of the contact-circle on which the three feet stand must be $44 \cdot 71$ millimetres. For, by reference to fig. 1, it will be seen that the radius $\mathrm{PM}$ of the contact-circle is such that

$$
(\mathrm{PM})^{2}=\mathrm{AM} \cdot \mathrm{MB}=2 r s-s^{2},
$$

where $s$ is the sagitta of the curve. Hence, if $r=1000$ millimetres, and $s=1$ millimetre, $P M=\sqrt{1999}=44 \cdot 7102$. The screw itself is cut on a steel tube 8 millimetres in diameter. The tips of the three feet and of the central point are of phosphor-bronze, which does not scratch glass. The arms carrying the three feet are girder-shaped, thin, deep ; so as to be very firm vertically; lateral flexure is immaterial. The tips of the three feet are adjusted by filing to chisel-shaped edges, forming little tangents to the imaginary circle of contact. The divided circle is not, as ordinarily, cut upon a flat plate, but is cut upon a thin cylinder of aluminium-bronze affixed to the screw by a light spider with three arms. The whole instrument weighs only 78 grammes. It was constructed for the author by Messrs. Nalder Brothers.

The formula for the focal power of any lens (formula no. (11), p. 244) consists of two factors-one depending solely on the shape of the lens, the other upon its material. The latter factor, $\frac{1-h}{h}$, is a mere numeric; whilst the 
former, being the difference of two curvatures, is itself a curvature. If the curvature thus determined by shape solely is expressed in dioptries, then, on multiplying by the numeric which depends on the nature of the material, the resultant power of the lens will also be expressed directly in dioptries. The dioptrie spherometer facilitates this desirable end by its directness of reading, quite apart from any incidental advantages which may result from the details of construction.

XXXIV. On the Mechanics of Luminosity.

By E. Wiedemann.

[Continued from p. 163.]

Experimental Measurements.

17. T $\mathrm{N}$ order to show how many of the questions raised in 1 the foregoing admit of an experimental solution I have made a number of measurements in various directions. The method of investigation is briefly the following :-

Since in all measurements the coefficients of emission are to be expressed in calorimetric measure, $i . e$. in gramme-calories per second, the numbers given by the amyl-acetate lamp, serving as the source of light, for comparison at a given distance from the photometer-slit must be reduced to absolute intensity. This was done by comparison with the radiation of a glowing platinum wire. From these measurements we obtain at once the energy in gramme-calories per second radiated by $1 \mathrm{gr}$. platinum, and, consequently, relationships between the total energy and that radiated in a definite region of the spectrum, e.g. in the yellow. Next, the total brightness of gas-flame coloured with sodium was compared with the brightness of the amyl-acetate lamp in the yellow ; and hence the coefficient of emission was calculated in grammecalories per second for one gramme and for a molecule of sodium.

Then follows the determination of the quantity $b$, and from its value that of the store of luminous energy $L$, and therefore of the kinetic energy of the luminous motions.

\section{Dependence of the Radiation of a Platinum Wire upon its Temperature.}

18. The measurements were carried out in the first place for the D-line. The amyl-acetate lamp was always employed as the comparison-lamp.

The arrangement followed in these measurements and for 